\title{
Aldosterone and Inflammation
}

\author{
Kimberly C. Gilbert, MD and Nancy J. Brown, MD
}

\begin{abstract}
Purpose of Review-Aldosterone causes tissue inflammation leading to fibrosis and remodeling in the heart, vasculature and kidney. We summarize recent data regarding the mechanism(s) through which aldosterone stimulates inflammation.
\end{abstract}

Recent Findings-Studies elucidate the cell-specific effects of mineralocorticoid receptor activation on inflammatory cell infiltration and adhesion, and highlight the role of the macrophage in the development of vascular collagen deposition and hypertension. Activation of NF- $\kappa \mathrm{B}$ in vascular smooth muscle cells involves a complex interplay between the angiotensin $\mathrm{AT}_{1}$ receptor and the mineralocorticoid receptor. Activation of the mineralocorticoid receptor by aldosterone stimulates an inflammatory phenotype in adipocytes and contributes to insulin resistance by increasing oxidative stress.

Summary-Mechanistic studies of aldosterone-induced inflammation provide the rationale an expanded therapeutic role for mineralocorticoid receptor antagonists and aldosterone synthase inhibitors.

\section{Keywords}

aldosterone; inflammation; reactive oxygen species; fibrosis

\section{Introduction}

Aldosterone binds to the mineralocorticoid receptor (MR) of principal cells in the collecting duct of the kidney, stimulates expression of genes such as serum- and glucocorticoidinducible kinase 1 (SGK1), and activates the epithelial sodium channel (ENaC) to regulate salt excretion, extracellular volume, and blood pressure. Aldosterone can exert effects in non-epithelial cells such as cardiomyocytes, endothelial cells, vascular smooth muscle cells (VSMC), mesangial cells and podocytes via the MR and subsequent genomic events, as well as through nongenomic pathways. Cortisol also binds the MR with high affinity. In epithelial tissues, the enzyme 11ß-hydroxysteroid dehydrogenase type 2 (11ßHSD-2) converts cortisol (or corticosterone in rodents) to cortisone, which does not bind MR. Many non-epithelial cells lack 11ßHSD-2 and thus cortisol occupies the MR of these cells. Studies conducted over the last 15 years reveal that aldosterone causes inflammation leading to fibrosis and remodeling in the heart, vasculature and kidney. Here we review recent

Corresponding Author: Nancy J. Brown, M.D., Robert H. Williams Professor of Medicine and Pharmacology, Vanderbilt University School of Medicine, Departments of Medicine and Clinical Pharmacology, Room 536 Robinson Research Building, Nashville, TN 37232-6602, nancy.j.brown@ vanderbilt.edu, P 615-343-8701, F 615-343-2551. 
developments in our understanding of the mechanism(s) through which aldosterone stimulates inflammation.

\section{Aldosterone induces inflammation by stimulating the formation of reactive oxygen species}

The generation of reactive oxygen species (ROS) such as superoxide and hydrogen peroxide triggers activation of proinflammatory transcription factors activator protein (AP)-1 and nuclear factor $(\mathrm{NF})-\kappa \mathrm{B}$. Systemic administration of aldosterone increases nicotinamide adenine dinucleotide phosphate [NADP $(\mathrm{H})]$ oxidase and oxidative stress in macrophages, the heart, vasculature, and kidney.[1-4] Aldosterone also decreases the expression of glucose-6-phosphate dehydrogenase (G6PD), which reduces NADP+ to NADPH.[5] Exogenous aldosterone stimulates aortic expression of NOX2 (also known as gp91phox) and p22phox through an MR-dependent mechanism and of p47phox mRNA through both $\mathrm{AT}_{1}$ receptor and MR-dependent mechanisms.[3] MR activation contributes to Ang II-mediated activation of NADPH oxidase in the heart and aorta.[6-8] Aldosterone stimulates activation of NF- $\kappa \mathrm{B}$ in the heart, an effect that is prevented in NOX 2 deficient mice.[7] Conversely, anti-oxidant drugs[1,3,9-11] decrease inflammation and injury in aldosterone-treated rodents.

Activation of NF- $\kappa \mathrm{B}$ by aldosterone further induces the production of adhesion molecules, chemokines such as monocyte chemoattractant protein (MCP-1), and inflammatory cytokines. In vitro aldosterone upregulates the lymphocyte chemoattractant factor IL-16, cytotoxic T-lymphocyte-associated protein 4 (CTLA4), and type I and type III collagens in human VSMCs.[12] Physiologic concentrations of aldosterone induce rapid increases in expression of genes involved in inflammation and fibrosis, including orosomucoid-1 (orm-1), plasminogen activator inhibitor-1 (PAI-1) and tenascin-X in MR-expressing cardiomyocytes, and PAI-1 in monocytes, mesangial cells, endothelial cells, and VSMCs. [13-16]

In vivo, treatment of rats with aldosterone and salt increases the expression of intercellular adhesion molecule (ICAM), cyclooxygenase (COX)-2, osteopontin, and MCP-1 in the heart and causes inflammatory arterial lesions with perivascular macrophages.[17] MR blockade decreases this inflammatory response. Aldosterone/salt treatment also causes MR-dependent perivascular leukocyte infiltration and increased expression of osteopontin, MCP-1, IL-6, and IL-1 $\beta$ in the kidney.[18] MR antagonism decreases aortic inflammation, fibrosis and hypertrophy in hypertensive rats,[19-21] and decreases oxidative stress and inflammation in apolipoprotein E-deficient mice fed a high-cholesterol diet, a model of atherosclerosis.[22]

\section{Endothelial MR regulates inflammatory cell infiltration and adhesion}

In addition to stimulating the formation of ROS, aldosterone may promote vascular inflammation by stimulating endothelial exocytosis and adhesion. Endothelial cells express functional MR as well as 11ßHSD-2.[23] Aldosterone stimulates endothelial expression of ICAM-1 and promotes the adhesion of leukocytes to endothelial cells.[23] Jeong et al demonstrated that physiological aldosterone stimulates the release of von Willebrand factor and IL-18, but not tissue plasminogen activator (t-PA), from human aortic endothelial cells. [24] At the same time, aldosterone enhanced the adherence of leukocytes to endothelial cells 
through a P-selectin-dependent mechanism. The group further demonstrated that aldosterone stimulates endothelial exocytosis through a nongenomic, but MR-dependent mechanism. That is, aldosterone stimulated endothelial exocytosis within 10 minutes and exocytosis was not blocked by actinomycin. Either spironolactone or anti-MR siRNA blocked aldosteronestimulated exocytosis.

Neutrophils also express MR and 11ßHSD-2. In neutrophils cultured on fibronectin, aldosterone inhibits the activation of NF- $\mathrm{kB}$ by IL-8 or granulocyte colony-stimulating factor, decreases expression of ICAM-1, and prevents adhesion through an MR-dependent mechanism.[25] Aldosterone does not affect and lipopolysaccharide-stimulated activation of NF- $\kappa B$ in neutrophils on fibronectin. Thus, the MR-dependent proinflammatory effects of aldosterone depend on the stimulus and target cell.

\section{Aldosteronethe promotes inflammation via MR-dependent and -independent mechanisms}

Activation of MR by aldosterone results in its dissociation from molecular chaperones, translocation into the nucleus and binding to hormone response elements in the regulatory region of target gene promoters to enhance expression. As noted earlier, aldosterone can also exert rapid, nongenomic effects. Data are conflicting as to whether or not these rapid effects occur via MR activation. For example, some rapid effects of aldosterone cannot be blocked by the MR antagonist spironolactone, but may be blocked by its open-ring water-soluble metabolite, canrenoate, or by eplerenone. [26,27] Studies in fibroblasts derived from MRdeficient mice and in MR-transfected human embryonic kidney (HEK) cells suggest that the MR contributes to the nongenomic effects of aldosterone on the extracellular signalregulated kinases 1 and 2 (ERK1/2) and c-Jun $\mathrm{NH}_{2}$-terminal kinase (JNK) pathway, but not to rapid effects on calcium. $[28,29]$ In rabbit heart, aldosterone increases $\mathrm{Na}^{+} / \mathrm{K}^{+} / 2 \mathrm{Cl}^{-}$cotransporter activity and decreases $\mathrm{Na}^{+} / \mathrm{K}^{+}$pump activity through a nongenomic effect on protein kinase $\mathrm{C}$-epsilon (PKCE),[30] which stimulates NFkB activation via MAPKs.[31] In addition, nongenomic effects of aldosterone may facilitate classical MR-mediated effects.

In VSMCs, phosphorylation or activation of p38, MAPKs, and ERK1/2 results in the production of cytokines and chemokines.[32] Aldosterone rapidly activates ERK1/2 in VSMCs.[33] Aldosterone also enhances both the rapid and the delayed activation of ERK1/2 by Ang II. The MR blocker, spironolactone does not block rapid phosphorylation of ERK1/2 by aldosterone.[34] In contrast, spironolactone or inhibitors of transcription and protein synthesis prevent the late effect of aldosterone and Ang II. The early phase involves the transactivation of the epidermal growth factor (EGF) receptor, whereas the late phase involves increased expression of the fibrotic and proliferative small and monomeric GTPbinding protein Ki-ras2A and mitogen activated protein kinase (MAPK).[33] In human aortic smooth muscle cells, aldosterone induces EGFR expression via an interaction between the ligand-bound MR and regions 316-163 and 163-1 bp of the EGFR promoter.[35]

\section{Crosstalk between the $\mathrm{AT}_{1}$ receptor and $\mathrm{MR}$ in vascular smooth muscle cells involved in the stimulation of pro-inflammatory pathways}

Just as aldosterone enhances the effects of $\mathrm{AT}_{1}$ activation, angiotensin II (Ang II) activates MR responsive elements through an $\mathrm{AT}_{1}$-dependent mechanism in human coronary artery 
smooth muscle cells.[12] Schiffrin and colleagues have further characterized the interactive effects of the $\mathrm{AT}_{1}$ and MRs by studying the effect of Ang II or aldosterone in mouse VSMCs transfected with a small interfering (si)RNA targeting $\mathrm{AT}_{1 \mathrm{a}}, \mathrm{AT}_{1 \mathrm{~b}}$ or MR or a negative control siRNA.[36] (The mouse has two $\mathrm{AT}_{1}$ receptors, $\mathrm{AT}_{1 \mathrm{a}}$ and $\mathrm{AT}_{1 \mathrm{~b}}$.) $\mathrm{AT}_{1 \mathrm{a}}$ receptor knockdown prevented aldosterone-induced phosphorylation of the proinflammatory ERK1/2, JNK, and NF- $\kappa \mathrm{B}$, as well as c-fos expression. Knockdown of the $\mathrm{AT}_{1 \mathrm{~b}}$ receptor also decreased aldosterone-induced activation of $\mathrm{NF}-\kappa \mathrm{B}$, but did not affect phosphorylation of ERK1/2 or JNK. MR knockdown abolished activation and nuclear translocation of NF$\kappa \mathrm{B}$ in response to either Ang II or aldosterone. In contrast, MR knockdown did not prevent Ang II- or aldosterone-induced phosphorylation of ERK1/2 or JNK. The MR antagonist eplerenone decreased phosphorylation of JNK in response to aldosterone and activation of $\mathrm{NF}-\kappa \mathrm{B}$ in response to either Ang II or aldosterone. Taken together, these data suggest that activation of NF- $\kappa \mathrm{B}$ by either Ang II or aldosterone requires both the $\mathrm{AT}_{1}$ receptor and the MR. Ang II can induce activation of ERK1/2 or JNK through $\mathrm{AT}_{1}$, in the absence of the MR. Aldosterone also stimulates activation of ERK1/2 through an $\mathrm{AT}_{1}$-dependent, MRindependent mechanism, but may require both $\mathrm{AT}_{1}$ and $\mathrm{MR}$ to activate JNK. Given that activation of ERK1/2 and JNK promotes vascular inflammation, these data suggest that $\mathrm{AT}_{1}$ receptor blockers and MR antagonists could have synergistic anti-inflammatory effects.

\section{Cell-specific pro-inflammatory effects of aldosterone in the kidney and retina}

Studies by Leroy and co-workers provide an interesting link between the proinflammatory and profibrotic effects of aldosterone in non-epithelial cells to the classic physiologic role of aldosterone in promoting epithelial sodium transport.[37] The investigators demonstrated that aldosterone activates the canonical NFא-B pathway and stimulates the expression of proinflammatory genes in principal cells, the site of MR-mediated effects of aldosterone on sodium reabsorption and potassium excretion. In the principal cell, aldosterone stimulates $\mathrm{NF}-\kappa \mathrm{B}$ via an MR-dependent increase in SGK1 expression rather than via ERK or p38. Interestingly, activation of NF- $\kappa \mathrm{B}$ by lipopolysaccharide (LPS) decreases SGK1 expression, activity of the ENaC a subunit, and glucocorticoid- and mineralocorticoid-stimulated sodium transport in cultured principal cells, [38] as well as amiloride-sensitive ion fluxes in cortical collecting duct cells.[39] Increased expression of the NF- $\kappa$ B target gene PAI-1 could also decrease activation of $\mathrm{ENaC}$ by decreasing plasmin-mediated proteolytic cleavage of its $\gamma$ subunit.[40] These data suggest that the proinflammatory effects of aldosterone may serve to modulate the effects of aldosterone on salt reabsorption.

Hypertension affects the microvasculature of the kidney and retina through many common mechanisms. Wilkinson-Berka et al. investigated the contribution of aldosterone and MR activation to pathological angiogenesis in rats with oxygen-induced retinopathy.[41] The authors identified aldosterone synthase, a functional MR, and 11 $\beta$ HSD-2 in the retina. Spironolactone reduced angiogenesis in this model. Treatment of rats with aldosterone and high salt intake caused increased leukocyte infiltration and angiogenesis in the retina, as well as increased retinal expression of NOX4 and MCP-1 and decreased retinal expression of G6PD. High salt alone also increased inflammation and angiogenesis and this effect was prevented by MR antagonism. Since inflammation leads to retinal vascular growth,[42] these studies suggest that MR antagonism could be effective in the prevention of diabetic 
retinopathy. Further studies are needed to assess the effect of MR antagonism on retinopathy in patients with diabetes.

\section{A key role for the macrophage in aldosterone-induced oxidative stress and remodeling}

Two recent studies highlight the critical role of the macrophage in aldosterone-induced inflammation. In the first, Schiffrin and colleagues reported that mice with reduced monocyte/macrophage-induced inflammation resulting from a mutation in the gene encoding macrophage-colony stimulating factor are protected from aldosterone/salt-induced oxidative stress and endothelial dysfunction.[43] Aortic collagen content was also decreased in monocyte/macrophage-impaired mice relative to wild-type (WT). Aldosterone increased IL-6 and IL-10 concentrations in monocyte/macrophage-impaired mice, suggesting that these cytokines can originate from T-lymphocytes in the absence of increased oxidative stress. Monocyte/macrophage impaired mice were not protected against aldosterone/saltinduced arterial fibronectin deposition or arterial stiffness.

Rickard et al reported on the effect of macrophage-specific deletion of the MR on inflammation in mice that were treated with the mineralocorticoid deoxycorticosterone (DOC) for 8 days, 4 weeks or 8 weeks.[44] At eight days, MCP-1 concentrations and macrophage infiltration in the heart were similar in WT and macrophage-specific MRknockout mice, while DOC/salt-induced NOX2 and PAI-1 expression was decreased. Blood pressure was lower in the macrophage-specific MR knockout mice at 4 weeks and 8 weeks. Despite having lower blood pressure, the macrophage-specific MR knockout mice were not protected against cardiac hypertrophy and seemed to have renal hypertrophy even in the absence of DOC/salt. DOC/salt-induced interstitial and perivascular collagen deposition were reduced in the macrophage-specific MR knockouts compared to WT at 8 weeks. In short, macrophage-specific MR knockout mice were protected against fibrosis and hypertension even though there was no change in macrophage infiltration.

This study has several important implications. First, the study confirms that the macrophage MR does not control macrophage infiltration, consistent with the reports by Caprio et al and Jeong et al that indicates that endothelial cell MR promotes macrophage adhesion and infiltration.[23,24] Second, the study adds a new dimension to the emerging understanding that inflammation contributes to the pathogenesis of hypertension. Harrison and co-workers have demonstrated that $\mathrm{T}$ cells, and $\mathrm{T}$ helper (Th)-17 cells in particular, are requisite for the generation of Ang II- or DOCA/salt-induced hypertension.[45,46] IL-17 stimulates macrophages and other cells to produce cytokines and induces remodeling. Deletion of the MR on macrophages may disrupt this process.

Third, the study may help resolve apparently conflicting data based on a cardiomyocytecentric view of fibrosis. Cardiomyocytes, like macrophages, lack 11ßHSD-2. For this reason, under physiological conditions, cardiac MRs are occupied by cortisol or corticosterone. Cardiomyocyte-specific over-expression of 11ßHSD-2 causes fibrosis.[47] Pharmacological MR antagonism prevents cardiac fibrosis in this model, leading some to suggest that endogenous cortisol acts as an antagonist at the cardiomyocyte MR.

Cardiomyocyte-specific MR deletion does not alter fibrosis in this model, however.[48] It 
would be interesting to know whether macrophage-specific deletion of the MR protected $11 \beta$ HSD-2 transgenics from hypertrophy.

\section{Aldosterone contributes to inflammation in obesity}

Aldosterone concentrations correlate with body mass index. Increased ROS and inflammation due to MR activation may contribute to insulin resistance in obesity.[49] Treatment of cultured adipocytes with aldosterone increases expression of IL-6, PAI-1, chemerin and leptin.[50] In contrast, treatment of adipocytes with the glucocorticoid receptor specific agonist dexamethasone inhibits the expression of IL-6, MCP-1, tumor necrosis factor- $a$, chemerin and leptin. In addition, in adipocytes in which the glucocorticoid receptor was knocked down or out, corticosterone increased the gene expression IL-6 and MCP-1, indicating that glucocorticoid receptor activation opposes the proinflammatory effects of MR activation. MR expression is increased in the adipocytes of obese mice. MR antagonism decreases the production of ROS, inflammatory gene expression and macrophage infiltration in adipose tissues and reduces insulin resistance in obese mice. $[51,52]$

\section{Aldosterone synthase inhibitors decrease inflammation in rodent models}

MR antagonists prevent the inflammatory effects of aldosterone in most rodent models. Aldosterone synthase inhibitors are also under development. However, since MR can be activated by ligands other than aldosterone, it does not necessarily follow that reducing aldosterone concentrations will reduce inflammation. Conversely, reducing aldosterone may offer advantages since MR antagonism does not block some rapid proinflammatory effects of aldosterone and even promotes activation of NF- $\kappa \mathrm{B}$ in neutrophils. Studies in aldosterone synthase deficient mice have begun to reveal tissue-specific contributions of endogenous aldosterone proinflammatory gene expression.[53] Pharmacological aldosterone synthase inhibition decreases cardiac and renal injury to the same extent as MR antagonism[54,55]. Inhibition of aldosterone synthase in the brain also decreases blood pressure.[56] The specificity of pharmacological inhibitors for aldosterone synthase, the enzyme responsible for the final step in the synthesis of aldosterone, versus $11 \beta$-hydroxylase, the enzyme responsible for the final step in the synthesis of cortisol, may be a challenge for drug development.[57] Aldosterone synthase inhibitors are under investigation for the treatment of hyperaldosteronism but could prevent end-organ damage in essential hypertension.

\section{Conclusion}

Studies published in the last year have elucidated cell-specific MR-dependent effects of aldosterone on cell adhesion and cytokine expression, as well as on renal function. The macrophage plays a central role in mineralocorticoid-induced vascular and cardiac fibrosis. Aldosterone contributes to the inflammatory phenotype of obesity and the induction of ROS by aldosterone also contributes to insulin resistance. Aldosterone synthase inhibitors, like MR antagonists, reduce inflammation. Inflammation contributes to vascular, cardiac and renal injury. Ongoing and future clinical trials will reveal the extent to which prevent the proinflammatory effects of aldosterone reduces end-organ damage. 


\section{Acknowledgments}

Dr. Brown serves as a consultant for Novartis and Merck. She receives grant support from Shire HRT and Forest Pharmaceuticals.

Disclosures: Funded in part by National Institutes of Health R01HL060906 and HL077389

\section{References}

1. Shibata S, Nagase M, Yoshida S, et al. Podocyte as the target for aldosterone: roles of oxidative stress and Sgk1. Hypertension. 2007; 49:355-364. [PubMed: 17200434]

2. Keidar S, Kaplan M, Pavlotzky E, et al. Aldosterone administration to mice stimulates macrophage NADPH oxidase and increases atherosclerosis development: a possible role for angiotensinconverting enzyme and the receptors for angiotensin II and aldosterone. Circulation. 2004; 109:2213-2220. [PubMed: 15123520]

3. Hirono Y, Yoshimoto T, Suzuki N, et al. Angiotensin II receptor type 1-mediated vascular oxidative stress and proinflammatory gene expression in aldosterone-induced hypertension: the possible role of local renin-angiotensin system. Endocrinology. 2007; 148:1688-1696. [PubMed: 17218415]

4. Nishiyama A, Yao L, Nagai Y, et al. Possible contributions of reactive oxygen species and mitogenactivated protein kinase to renal injury in aldosterone/salt-induced hypertensive rats. Hypertension. 2004; 43:841-848. [PubMed: 14769808]

5. Leopold JA, Dam A, Maron BA, et al. Aldosterone impairs vascular reactivity by decreasing glucose-6-phosphate dehydrogenase activity. Nat.Med. 2007; 13:189-197. [PubMed: 17273168]

6. Virdis A, Neves MF, Amiri F, et al. Spironolactone improves angiotensin-induced vascular changes and oxidative stress. Hypertension. 2002; 40:504-510. [PubMed: 12364354]

7. Johar S, Cave AC, Narayanapanicker A, et al. Aldosterone mediates angiotensin II-induced interstitial cardiac fibrosis via a Nox2-containing NADPH oxidase. FASEB J. 2006; 20:1546-1548. [PubMed: 16720735]

8. Stas S, Whaley-Connell A, Habibi J, et al. Mineralocorticoid receptor blockade attenuates chronic overexpression of the renin-angiotensin-aldosterone system stimulation of reduced nicotinamide adenine dinucleotide phosphate oxidase and cardiac remodeling. Endocrinology. 2007; 148:37733780. [PubMed: 17494996]

9. Iglarz M, Touyz RM, Viel EC, et al. Involvement of oxidative stress in the profibrotic action of aldosterone. Interaction with the renin-angiotension system. Am.J.Hypertens. 2004; 17:597-603. [PubMed: 15243979]

10. Sun Y, Zhang J, Lu L, et al. Aldosterone-induced inflammation in the rat heart : role of oxidative stress. Am.J.Pathol. 2002; 161:1773-1781. [PubMed: 12414524]

11. Park YM, Park MY, Suh YL, et al. NAD(P)H oxidase inhibitor prevents blood pressure elevation and cardiovascular hypertrophy in aldosterone-infused rats. Biochem.Biophys.Res.Commun. 2004; 313:812-817. [PubMed: 14697264]

12. Jaffe IZ, Mendelsohn ME. Angiotensin II and aldosterone regulate gene transcription via functional mineralocortocoid receptors in human coronary artery smooth muscle cells. Circ.Res. 2005; 96:643-650. [PubMed: 15718497]

13. Fejes-Toth G, Naray-Fejes-Toth A. Early aldosterone-regulated genes in cardiomyocytes: clues to cardiac remodeling? Endocrinology. 2007; 148:1502-1510. [PubMed: 17234708]

14. Yuan J, Jia R, Bao Y. Aldosterone up-regulates production of plasminogen activator inhibitor-1 by renal mesangial cells. J Biochem.Mol.Biol. 2007; 40:180-188. [PubMed: 17394767]

15. Brown NJ, Kim KS, Chen YQ, et al. Synergistic effect of adrenal steroids and angiotensin II on plasminogen activator inhibitor-1 production. J.Clin.Endocrinol.Metab. 2000; 85:336-344. [PubMed: 10634408]

16. Calo LA, Zaghetto F, Pagnin E, et al. Effect of aldosterone and glycyrrhetinic acid on the protein expression of PAI-1 and p22(phox) in human mononuclear leukocytes. J.Clin.Endocrinol.Metab. 2004; 89:1973-1976. [PubMed: 15070972] 
17. Rocha R, Rudolph AE, Frierdich GE, et al. Aldosterone induces a vascular inflammatory phenotype in the rat heart. Am.J.Physiol Heart Circ.Physiol. 2002; 283:H1802-H1810. [PubMed: 12384457]

18. Blasi ER, Rocha R, Rudolph AE, et al. Aldosterone/salt induces renal inflammation and fibrosis in hypertensive rats. Kidney Int. 2003; 63:1791-1800. [PubMed: 12675855]

19. Benetos A, Lacolley P, Safar ME. Prevention of aortic fibrosis by spironolactone in spontaneously hypertensive rats. Arterioscler.Thromb.Vasc.Biol. 1997; 17:1152-1156. [PubMed: 9194767]

20. Lacolley P, Labat C, Pujol A, et al. Increased carotid wall elastic modulus and fibronectin in aldosterone-salt-treated rats: effects of eplerenone. Circulation. 2002; 106:2848-2853. [PubMed: 12451013]

21. Neves MF, Amiri F, Virdis A, et al. Role of aldosterone in angiotensin II-induced cardiac and aortic inflammation, fibrosis, and hypertrophy. Can.J.Physiol Pharmacol. 2005; 83:999-1006. [PubMed: 16391708]

22. Suzuki J, Iwai M, Mogi M, et al. Eplerenone with valsartan effectively reduces atherosclerotic lesion by attenuation of oxidative stress and inflammation. Arterioscler.Thromb.Vasc.Biol. 2006; 26:917-921. [PubMed: 16424347]

23. Caprio M, Newfell BG, la SA, et al. Functional mineralocorticoid receptors in human vascular endothelial cells regulate intercellular adhesion molecule-1 expression and promote leukocyte adhesion. Circ.Res. 2008; 102:1359-1367. [PubMed: 18467630]

24*. Jeong Y, Chaupin DF, Matsushita K, et al. Aldosterone activates endothelial exocytosis. Proc.Natl.Acad.Sci.U.S.A. 2009; 106:3782-3787. [PubMed: 19223584] Study shows that aldosterone stimulates exocytosis of adhesion factors from endothelial cells via the MR.

25**. Bergmann A, Eulenberg C, Wellner M, et al. Aldosterone abrogates nuclear factor kappaBmediated tumor necrosis factor alpha production in human neutrophils via the mineralocorticoid receptor. Hypertension. 2010; 55:370-379. [PubMed: 20065153] This is the first study to demonstrate an anti-inflammatory effect of MR activation by aldosterone in the neutrophil.

26. Michea L, Delpiano AM, Hitschfeld C, et al. Eplerenone blocks nongenomic effects of aldosterone on the $\mathrm{Na}+\mathrm{H}+$ exchanger, intracellular $\mathrm{Ca} 2+$ levels, and vasoconstriction in mesenteric resistance vessels. Endocrinology. 2005; 146:973-980. [PubMed: 15550504]

27. Boldyreff B, Wehling M. Aldosterone: refreshing a slow hormone by swift action. News Physiol Sci. 2004; 19:97-100. [PubMed: 15143201]

28. Haseroth K, Gerdes D, Berger S, et al. Rapid nongenomic effects of aldosterone in mineralocorticoid-receptor-knockout mice. Biochem.Biophys.Res.Commun. 1999; 266:257-261. [PubMed: 10581199]

29. Grossmann C, Benesic A, Krug AW, et al. Human mineralocorticoid receptor expression renders cells responsive for nongenotropic aldosterone actions. Mol.Endocrinol. 2005; 19:1697-1710. [PubMed: 15761031]

30. Mihailidou AS, Mardini M, Funder JW. Rapid, nongenomic effects of aldosterone in the heart mediated by epsilon protein kinase C. Endocrinology. 2004; 145:773-780. [PubMed: 14605011]

31. Li RC, Ping P, Zhang J, et al. PKCepsilon modulates NF-kappaB and AP-1 via mitogen-activated protein kinases in adult rabbit cardiomyocytes. Am.J.Physiol Heart Circ.Physiol. 2000; 279:H1679-H1689. [PubMed: 11009455]

32. Mukundan L, Milhorn DM, Matta B, et al. CD40-mediated activation of vascular smooth muscle cell chemokine production through a Src-initiated, MAPK-dependent pathway. Cell Signal. 2004; 16:375-384. [PubMed: 14687667]

33. Min LJ, Mogi M, Li JM, et al. Aldosterone and angiotensin II synergistically induce mitogenic response in vascular smooth muscle cells. Circ.Res. 2005; 97:434-442. [PubMed: 16081869]

34. Mazak I, Fiebeler A, Muller DN, et al. Aldosterone potentiates angiotensin II-induced signaling in vascular smooth muscle cells. Circulation. 2004; 109:2792-2800. [PubMed: 15159288]

35. Grossmann C, Krug AW, Freudinger R, et al. Aldosterone-induced EGFR expression: interaction between the human mineralocorticoid receptor and the human EGFR promoter. Am.J.Physiol Endocrinol.Metab. 2007; 292:E1790-E1800. [PubMed: 17311890]

$36^{* *}$. Lemarie CA, Simeone SM, Nikonova A, et al. Aldosterone-induced activation of signaling pathways requires activity of angiotensin type 1a receptors. Circ.Res. 2009; 105:852-859. 
[PubMed: 19762686] A workman-like dissection of the contribution of AT1 and MR activation to downstream signaling in vascular smooth muscle cells.

37*. Leroy V, de SS, Agassiz V, et al. Aldosterone activates NF-kappaB in the collecting duct. J Am.Soc.Nephrol. 2009; 20:131-144. [PubMed: 18987305] Highlights the artificial distinction between aldosterone's "classic" and proinflammatory effects

38. de Seigneux S, Leroy V, Ghzili H, et al. NF-kappaB inhibits sodium transport via down-regulation of SGK1 in renal collecting duct principal cells. J Biol.Chem. 2008; 283:25671-25681. [PubMed: 18586672]

39. Bens $\mathrm{M}$, Chassin $\mathrm{C}$, Vandewalle A. Regulation of $\mathrm{NaCl}$ transport in the renal collecting duct: lessons from cultured cells. Pflugers Arch. 2006; 453:133-146. [PubMed: 16937117]

40. Passero CJ, Mueller GM, Rondon-Berrios H, et al. Plasmin activates epithelial $\mathrm{Na}+$ channels by cleaving the gamma subunit. J Biol.Chem. 2008

$41 *$. Wilkinson-Berka JL, Tan G, Jaworski K, et al. Identification of a retinal aldosterone system and the protective effects of mineralocorticoid receptor antagonism on retinal vascular pathology. Circ.Res. 2009; 104:124-133. [PubMed: 19038868] Identifies role for aldosterone in retinopathy

42. Ishida S, Yamashiro K, Usui T, et al. Leukocytes mediate retinal vascular remodeling during development and vaso-obliteration in disease. Nat.Med. 2003; 9:781-788. [PubMed: 12730690]

43*. Leibovitz E, Ebrahimian T, Paradis P, et al. Aldosterone induces arterial stiffness in absence of oxidative stress and endothelial dysfunction. J Hypertens. 2009 One of two recent papers highlighting the importance of the macrophage in aldosterone-induced inflammation

44**. Rickard AJ, Morgan J, Tesch G, et al. Deletion of mineralocorticoid receptors from macrophages protects against deoxycorticosterone/salt-induced cardiac fibrosis and increased blood pressure. Hypertension. 2009; 54:537-543. [PubMed: 19635989] This work demonstrates that MR on the macrophage contribute to the development of mineralocorticoid-stimulated hypertension without influencing infiltration.

45. Guzik TJ, Hoch NE, Brown KA, et al. Role of the T cell in the genesis of angiotensin II induced hypertension and vascular dysfunction. J.Exp.Med. 2007; 204:2449-2460. [PubMed: 17875676]

46**. Madhur MS, Lob HE, McCann LA, et al. Interleukin 17 promotes angiotensin II-induced hypertension and vascular dysfunction. Hypertension. 2010; 55:500-507. [PubMed: 20038749] One of several seminal papers from this group defining the role of the T-lymphocyte in the pathogenesis of hypertension

47. Qin W, Rudolph AE, Bond BR, et al. Transgenic model of aldosterone-driven cardiac hypertrophy and heart failure. Circ.Res. 2003; 93:69-76. [PubMed: 12791709]

48. Qin WN, Bond BR, Donoho GP, et al. Mineralocorticoid receptor mediates heart failure in the 11 ss-hydroxysteroid dehydrogenase type 2 transgenic mouse model. Circulation. 2004; 110:2946. [PubMed: 15505105]

49. Lastra G, Whaley-Connell A, Manrique C, et al. Low-dose spironolactone reduces reactive oxygen species generation and improves insulin-stimulated glucose transport in skeletal muscle in the TG(mRen2)27 rat. Am.J Physiol Endocrinol.Metab. 2008; 295:E110-E116. [PubMed: 18445755]

50*. Hoppmann J, Perwitz N, Meier B, et al. The balance between gluco- and mineralo-corticoid action critically determines inflammatory adipocyte responses. J Endocrinol. 2010; 204:153-164. [PubMed: 19939912] Suggests that aldosterone provides a link between obesity and inflammation

51*. Hirata A, Maeda N, Hiuge A, et al. Blockade of mineralocorticoid receptor reverses adipocyte dysfunction and insulin resistance in obese mice. Cardiovasc.Res. 2009; 84:164-172. [PubMed: 19505930] Supports a role for MR antagonism in obesity

52. Guo C, Ricchiuti V, Lian BQ, et al. Mineralocorticoid receptor blockade reverses obesity-related changes in expression of adiponectin, peroxisome proliferator-activated receptor-gamma, and proinflammatory adipokines. Circulation. 2008; 117:2253-2261. [PubMed: 18427128]

53*. Luther JM, Wang Z, Ma J, et al. Endogenous aldosterone contributes to acute angiotensin IIstimulated plasminogen activator inhibitor- 1 and preproendothelin- 1 expression in heart but not aorta. Endocrinology. 2009; 150:2229-2236. [PubMed: 19106220] Creative use of aldosterone synthase-deficient mice to probe proinflammatory effects of endogenous aldosterone 
54. Fiebeler A, Nussberger J, Shagdarsuren E, et al. Aldosterone synthase inhibitor ameliorates angiotensin II-induced organ damage. Circulation. 2005; 111:3087-3094. [PubMed: 15939810]

55*. Lea WB, Kwak ES, Luther JM, et al. Aldosterone antagonism or synthase inhibition reduces endorgan damage induced by treatment with angiotensin and high salt. Kidney Int. 2009; 75:936944. [PubMed: 19225557] Provides a direct comparison between effects of MR antagonism and aldosterone synthase inhibition in angiotensin-induced hypertensive end-organ damage

56*. Gomez-Sanchez EP, Gomez-Sanchez CM, Plonczynski M, et al. Aldosterone synthesis in the brain contributes to Dahl salt-sensitive rat hypertension. Exp.Physiol. 2010; 95:120-130.

[PubMed: 19837774] Demonstrates that central aldosterone synthesis contributes to salt-sensitive hypertension

57*. LaSala D, Shibanaka Y, Jeng AY. Coexpression of CYP11B2 or CYP11B1 with adrenodoxin and adrenodoxin reductase for assessing the potency and selectivity of aldosterone synthase inhibitors. Anal.Biochem. 2009; 394:56-61. [PubMed: 19622340] Addresses strategy for the development of specific aldosterone synthase inhibitors 